PROCEEDINGS OF THE

AMERICAN MATHEMATICAL SOCIETY

Volume 130, Number 4, Pages 999-1004

S 0002-9939(01)06313-4

Article electronically published on November 9, 2001

\title{
REGULARITY OF VISCOSITY SOLUTIONS OF A DEGENERATE PARABOLIC EQUATION
}

\author{
YUN-GUANG LU AND LIWEN QIAN
}

(Communicated by Suncica Canic) \begin{abstract}
bolic equation of second order

$$
\begin{cases}u_{t}=u \triangle u-\gamma|\nabla u|^{2} & \text { in } \Omega=R^{N} \times R^{+}, \\ u(x, 0)=u_{0}(x) & \text { in } \quad R^{N}\end{cases}
$$
\end{abstract}

ABstract. We study the Cauchy problem for the nonlinear degenerate para-

and present regularity results for the viscosity solutions.

\section{IntroduCtion}

We study the Cauchy problem for the equation

$$
u_{t}=u \triangle u-\gamma|\nabla u|^{2} \text { in } \Omega=R^{N} \times R^{+},
$$

with the initial data

$$
u(x, 0)=u_{0}(x) \quad \text { in } \quad R^{N} .
$$

Here $\gamma$ is a nonnegative constant and the initial function $u_{0}$ satisfies $u_{0} \in C\left(R^{N}\right) \cap$ $L^{\infty}\left(R^{N}\right)$ and $u_{0} \geq 0$ in $R^{N}$.

Equation (1) arises in several applications in biology and physics [1 2, 3]. It is degenerate parabolic at the points where $u$ vanishes. Therefore the Cauchy problem (1), (2), in general, has no classical solutions. The weak solutions are defined as follows:

Definition 1. A function $u \in L^{\infty}(\Omega) \cap L_{L o c}^{2}\left([0,+\infty] ; H_{L o c}^{1}\left(R^{N}\right)\right)$ is called a weak solution of (1), (2) if $u \geq 0$ almost everywhere in $\Omega$ and

$$
\int_{R^{N}} u_{0} \psi(0) d x+\iint_{\Omega}\left(u \psi_{t}-u \nabla u \cdot \nabla \psi-(1+\gamma)|\nabla u|^{2} \psi\right) d x d t=0
$$

for any $\psi \in C^{1,1}(\bar{\Omega})$ with compact support in $\bar{\Omega}$.

In [2] a weak solution is constructed by the well-known viscosity method: let $\omega_{\epsilon}(x, t)$ be the unique solution in $C^{2,1}(\Omega) \cap C(\bar{\Omega}) \cap L^{\infty}(\Omega)$ of the Cauchy problem with equation (1) replaced by

$$
u_{t}=u \triangle u-\gamma|\nabla u|^{2}+\epsilon \triangle u
$$

Received by the editors November 1, 1998 and, in revised form, April 10, 2000.

2000 Mathematics Subject Classification. Primary 35K55; Secondary 35K65, 35D10.

Key words and phrases. Degenerate parabolic equation, viscosity solution, Lipschitz continuity, maximum principle. 
where $\epsilon>0$ (see [4]). Then

$$
u_{\epsilon}=\omega_{\epsilon}+\epsilon
$$

is a classical solution of the Cauchy problem with $u_{0}$ replaced by $u_{0_{\epsilon}}=u_{0}+\epsilon$. Since $u_{\epsilon}(x, t)$ is nonincreasing with respect to $\epsilon$,

$$
u(x, t)=\lim _{\epsilon \backslash 0} u_{\epsilon}(x, t)=\lim _{\epsilon \searrow 0} \omega_{\epsilon}(x, t)
$$

is well definded for all $(x, t) \in \bar{\Omega}$.

It is proved in [2] that $u$ is a weak solution of (1), (2).

Definition 2. The weak solution of (1), (2) constructed by the vanishing viscosity method is called the viscosity solution.

Let $u(x, t)$ be the viscosity solution of (1), (2). Then it is proved in [2] that

$$
N=1 \Rightarrow u \in C(\bar{\Omega})
$$

and

$$
\gamma>\frac{1}{2} N \Rightarrow u \in C(\bar{\Omega}) \cap C^{1,1}(\Omega) .
$$

So, it is natural to ask what the smoothness of $u$ is if $N \geq 2$ and $0 \leq \gamma \leq \frac{N}{2}$. One result in 2 shows that if

$$
N \geq 2,0 \leq \gamma<1,
$$

then $u$ is not necessarily continuous in $\bar{\Omega}$.

In this paper, by using the method developed in [5, 6, 7], we study the regularity of the viscosity solution of the Cauchy problem (1), (2) for $\gamma \geq \sqrt{2 N}-1$, and obtain certain conditions under which $u(x, t)$ is Lipschitz continuous. We also show that for $\gamma<\sqrt{2 N}-1$ this method will meet difficulty except for special cases.

The method is to apply the maximum principle to obtain uniform estimates after some suitable transforms to the original equations. A similar idea was also used by P.Z. Mkrtychyan to different problems. The readers may refer to the references [8], 9] for the details.

\section{THEOREM AND PROOF}

The main result is the following.

Theorem. If $\gamma \geq \sqrt{2 N}-1,\left|\nabla\left(u_{0}^{1+\frac{\alpha}{2}}\right)\right| \leq M$, where $M$ is a positive constant, $\alpha^{2}+(\gamma+1) \alpha+\frac{N}{2} \leq 0$, then the viscosity solution $u(x, t)$ of equation (1), (2) satisfies $\left|\nabla\left(u^{1+\frac{\alpha}{2}}\right)\right| \leq M$ in $\bar{\Omega}$.

Proof. We may add a small positive perturbation $\epsilon$ to the initial data (2) and the "viscosity" term $\epsilon \triangle u$ to the right-hand side of equation (1), and then resolve the Cauchy problem for this new strictly parabolic equation with positive initial data and obtain the approximated solutions $u_{\epsilon}$. If $u_{\epsilon}$ satisfy some regular estimates, then there exists a subsequence $u_{\epsilon_{l}}$ converging uniformly as $\epsilon_{l} \rightarrow 0^{+}$, on any bounded region, to the solution $u$ of the Cauchy problem (12), (2). This technique is standard. For simplicity, we omit the details and only give the uniform estimate.

Let

$$
w=\frac{1}{2} \sum_{i=1}^{N} u_{x_{i}}^{2}
$$


From (7) we obtain that

$$
\begin{aligned}
w_{t} & =\sum_{i=1}^{N} u_{x_{i}}\left[u_{x_{i}} \Delta u+u\left(\sum_{j=1}^{N} u_{x_{i} x_{j} x_{j}}\right)-\gamma(2 w)_{x_{i}}\right] \\
& =2 w \Delta u+u \sum_{i, j=1}^{N}\left[\left(u_{x_{i}} u_{x_{i} x_{j}}\right)_{x_{j}}-u_{x_{i} x_{j}}^{2}\right]-2 \gamma \sum_{i=1}^{N} u_{x_{i}} w_{x_{i}} \\
& =2 w \Delta u+u \Delta w-u \sum_{i, j=1}^{N} u_{x_{i} x_{j}}^{2}-2 \gamma \sum_{i=1}^{N} u_{x_{i}} w_{x_{i}} .
\end{aligned}
$$

Set

$$
z=f(u) w
$$

Then

$$
w_{x_{i}}=\left(f^{-1}\right)_{x_{i}} z+f^{-1} z_{x_{i}}
$$

and

$$
w_{x_{i} x_{i}}=\left(f^{-1}\right)_{x_{i} x_{i}} z+2\left(f^{-1}\right)_{x_{i}} z_{x_{i}}+f^{-1} z_{x_{i} x_{i}} .
$$

These imply that

$$
\Delta w=f^{-1} \Delta z-2 f^{-2} f^{\prime} \sum_{i=1}^{N} u_{x_{i}} z_{x_{i}}+2\left(\frac{2 f^{\prime 2}-f f^{\prime \prime}}{f^{4}}\right) z^{2}-\frac{f^{\prime}}{f^{2}} z \Delta u .
$$

So from (81), (19) and (12) we get

$$
\begin{aligned}
z_{t} & =2 f(u) w \Delta u-u f(u) \sum_{i, j=1}^{N} u_{x_{i} x_{j}}^{2}+f^{\prime}(u)(u \Delta u-2 \gamma w) w \\
& +f(u) \Delta w-2 \gamma f(u) \sum_{i=1}^{N} u_{x_{i}} w_{x_{i}} \\
& =u \Delta z-\left(2 f^{-1} u f^{\prime}+2 \gamma\right) \sum_{i=1}^{N} u_{x_{i} z_{x_{i}}} \\
& +\left(\frac{4 u f^{\prime 2}}{f^{3}}-\frac{2 u f^{\prime \prime}}{f^{2}}+\frac{2 \gamma f^{\prime}}{f^{2}}\right) z^{2} \\
& +2 z \Delta u-u f(u) \sum_{i, j=1}^{N} u_{x_{i} x_{j}}^{2} .
\end{aligned}
$$

We choose in (13)

$$
f(u)=u^{\alpha} .
$$

Since

$$
\sum_{i, j=1}^{N} u_{x_{i} x_{j}}^{2} \geq \frac{1}{N}(\Delta u)^{2}
$$


then from (13), (14) and (15)

$$
\begin{aligned}
z_{t} \leq & u \Delta z-2(\alpha+\gamma) \sum_{i=1}^{N} u_{x_{i}} z_{x_{i}} \\
& +2 \alpha(\alpha+\gamma+1) u^{-\alpha-1} z^{2}+2 z \Delta u-\frac{u^{\alpha+1}}{N}(\Delta u)^{2}
\end{aligned}
$$

For $\gamma \geq \sqrt{2 N}-1$, if $\alpha$ satisfies

$$
\alpha^{2}+(\gamma+1) \alpha+\frac{N}{2} \leq 0
$$

then

$$
2 \alpha(\alpha+\gamma+1) u^{-\alpha-1} z^{2}+2 z \Delta u-\frac{u^{\alpha+1}}{N}(\Delta u)^{2} \leq 0 .
$$

Therefore from (16) and (18) we have

$$
z_{t} \leq u \Delta z-2(\alpha+\gamma) \sum_{i=1}^{N} u_{x_{i}} z_{x_{i}} .
$$

By applying the maximum principle to (19), we have $|z|_{\infty} \leq\left|z_{0}\right|_{\infty}$. From (7), (9) and (14) we get $\left|\nabla\left(u^{1+\frac{\alpha}{2}}\right)\right| \leq M$ provided $\left|\nabla\left(u_{0}^{1+\frac{\alpha}{2}}\right)\right| \leq M$ where $\alpha$ satisfies (17) and $M$ is a positive constant.

By the Theorem, we can easily draw some conclusions about the smoothness of $u$ if $\gamma \geq \sqrt{2 N}-1$.

Corollary. Under the same conditions as in the Theorem, the viscosity solution $u(x, t)$ of equations (11), (21) is Lipschitz continuous with respect to $x$ and Hölder continuous with exponent $\frac{1}{2}$ with respect to $t$ in $\bar{\Omega}$.

Proof. From the construction of the viscosity solution $u(x, t)$, we know that it can be approximated from above by the classical solution $u_{\epsilon}$. So from the Theorem,

$$
\left|u_{x_{i}}\right| \leq M u^{-\frac{\alpha}{2}}<M_{1},
$$

where $M_{1}>0$ is a constant.

The Hölder continuity in $t$ follows directly from a result by Gilding [10].

Remark 1 . Since $\sqrt{2 N}-1 \leq \frac{N}{2}$, the result improves the continuity result for $\gamma \geq \frac{N}{2}$ in [2].

Remark 2. The smoothness of $u$ if $0 \leq \gamma<\sqrt{2 N}-1$ remains an open problem. By the above approach it can only be solved for very special cases.

From (13) we find that if $f(u)$ satisfies

$$
u^{2} f f^{\prime \prime}-\gamma u f f^{\prime}-2 u^{2} f^{\prime 2} \geq \frac{N}{2} f^{2}
$$

then

$$
\left(\frac{4 u f^{\prime 2}}{f^{3}}-\frac{2 u f^{\prime \prime}}{f^{2}}+\frac{2 \gamma f^{\prime}}{f^{2}}\right) z^{2}+2 z \Delta u-u f(u) \sum_{i, j=1}^{N} u_{x_{i} x_{j}}^{2} \geq 0 .
$$


Thus we have from (13) and (22) that

$$
z_{t} \leq u \Delta z-\left(2 f^{-1} u f^{\prime}+2 \gamma\right) \sum_{i=1}^{N} u_{x_{i}} z_{x_{i}} .
$$

Applying the maximum principle to (23), we have $|z|_{\infty} \leq\left|z_{0}\right|_{\infty}$. Therefore we may have

$$
f(u) \sum_{i=1}^{N} u_{x_{i}}^{2} \leq M
$$

when

$$
f\left(u_{0}\right) \sum_{i=1}^{N} u_{0 x_{i}}^{2} \leq M .
$$

If we can find some other $f(u)$ satisfying (21), we may hope to get some new estimates. However, it is not successful. In the special case of (21) when it turns to be an equation, we may set

$$
F=\frac{d \ln f(u)}{d u}
$$

Then

$$
u^{2} F^{\prime}=u^{2} F^{2}+\gamma u F+\frac{N}{2} .
$$

Set again

$$
h(u)=u F(u) .
$$

It turns out to be

$$
\frac{d h}{\left(h+\frac{\gamma+1}{2}\right)^{2}+\frac{2 N-(\gamma+1)^{2}}{4}}=\frac{d u}{u} .
$$

If $\gamma<\sqrt{2 N}-1$, the solutions of (21) are given by

$$
f(u)=u^{-\frac{\gamma+1}{2}}|\cos (\alpha \ln u+c)|^{-1},
$$

where

$$
\alpha^{2}=\frac{2 N-(\gamma+1)^{2}}{4}
$$

But (25) can only be satisfied for very special $u_{0}$.

\section{ACKNOWLEDGEMENT}

The authors are very grateful to the editor and the referee for their many valuable suggestions, which greatly improved the main results in this paper. 


\section{REFERENCES}

1. M. Ughi, A Degenerate Parabolic Equation Modelling the Spread of an Epidemic, Ann. Mat. Pura Appl. 143 (1986), 385-400. MR 88g:35105

2. M. Bertsch, R.D. Passo, and M. Ughi, Discontinuous "viscosity" Solution of A Degenerate Parabolic Equation, Transactions of The American Mathematical Society 320 (1990), 2, 779798. MR 90m:35086

3. M. Bertsch and M. Ughi, Positivity properties of viscosity solutions of a degenerate parabolic equation, Nonlinear Anal. TMA. 14 (1990), 7, 571-592. MR 92a:35006

4. A. Friedman, Partial Differential Equations of Parabolic Type, Englewood Cliffs, N.J., Prentice-Hall Inc., 1964. MR 31:6062

5. Yunguang Lu, Hölder Estimates of Solutions of Biological Population Equations, Applied Mathematical Letters 13 (2000), 123-126. MR 2001b:35182

6. Yunguang $\mathrm{Lu}$, Hölder estimates of solutions to some doubly nonlinear degenerate parabolic equations, Commun. PDE. 24(5, 6) (1999), 895-913. MR 2000c:35105

7. Liwen Qian and Wentao Fan, Hölder estimates of Solutions for Some Degenerate Parabolic Equations, Acta Math. Sci. 4 (1999). MR 2000j:35156

8. P.Z. Mkrtychyan, A degenerate quasilinear parabolic equation that arises in the theory of nonstationary filtration, Izv. Akad. Nauk Armyan. SSSR Mat 24 (1989), 103-116 (English transl. in Soviet J. Contemp. Math. 24 (1989), 1-13). MR 90i:35134.

9. P.Z. Mkrtychyan, Estimation of the gradient of a solution and the classical solvability of the first initial-boundary value problem for a class of quasilinear nonuniformly parabolic equations, Izv. Akad. Nauk Armyan. SSSR Mat 24 (1989), 293-299 (English transl. in Soviet J. Contemp. Math. 24 (1989), 85-89). MR 90k:35141

10. B. H. Gilding, Hölder continuity of solutions of parabolic equations, J. London Math. Soc. 13 (1976), 103-106. MR 53:3501

Departamento de Matematicas y Estadistica, Universidad Nacional de Colombia, BoGOTA, Colombia

E-mail address: yglu@matematicas.unal.edu.co

Department of Computational Science, National University of Singapore, Singapore 117543

E-mail address: qianlw@cz3.nus.edu.sg

Current address: Singapore-MIT Alliance, National University of Singapore, Singapore 119260

E-mail address: smaqlw@nus.edu.sg 